\title{
On the local moduli of squareness
}

by

\author{
Antonio J. Guirao (Murcia)
}

\begin{abstract}
We introduce the notions of pointwise modulus of squareness and local modulus of squareness of a normed space $X$. This answers a question of C. Benítez, K. Przesławski and D. Yost about the definition of a sensible localization of the modulus of squareness. Geometrical properties of the norm of $X$ (Fréchet smoothness, Gâteaux smoothness, local uniform convexity or strict convexity) are characterized in terms of the behaviour of these moduli.
\end{abstract}

1. Introduction. Let us recall the notion of modulus of squareness, originally defined in [7], where it arose naturally from studying Lipschitz continuous set-valued functions. Given a normed space $X$, one observes that for any $x, y \in X$ with $\|y\|<1<\|x\|$, there is a unique $z=z(x, y)$ in the line segment $[x, y]$ with $\|z\|=1$. We put

$$
\omega(x, y)=\frac{\|x-z(x, y)\|}{\|x\|-1}
$$

and define $\xi=\xi_{X}:[0,1) \rightarrow[1, \infty]$ by

$$
\xi(\beta)=\sup \{\omega(x, y):\|y\| \leq \beta<1<\|x\|\} .
$$

It is shown in [7] that for an inner product space, $\xi(\beta)=\xi_{2}(\beta)=1 / \sqrt{1-\beta^{2}}$, and for any normed space containing $l_{1}(2), \xi(\beta)=\xi_{1}(\beta)=(1+\beta) /(1-\beta)$. The following theorem [1, Theorem $\mathrm{O}]$ puts together all the known properties of this modulus.

THEOREM 1.1. Let $X$ be any normed space, and $\xi$ its modulus of squareness. Then

(a) $\xi(\beta)=\sup \left\{\xi_{M}(\beta): M \subset X, \operatorname{dim} M=2\right\}$,

(b) $\xi$ is strictly increasing and convex,

2000 Mathematics Subject Classification: Primary 46B10, 46B20; Secondary 46B03.

Key words and phrases: Banach spaces, locally uniformly rotund norms, Fréchet smooth norms, strict convex norms, Gâteaux smooth norms, modulus of squareness.

Research supported by the grants MTM2005-08379 of MECD (Spain), 00690/PI/04 of Fundación Séneca (CARM, Spain) and AP2003-4453 of MECD (Spain). 
(c) $\xi<\xi_{1}$ everywhere on $(0,1)$, unless $X$ contains arbitrarily close copies of $l_{1}(2)$,

(d) $\xi^{\prime} \leq \xi_{1}^{\prime}$ almost everywhere on $(0,1)$,

(e) $\xi>\xi_{2}$ everywhere on $(0,1)$, unless $X$ is an inner product space,

(f) $X$ is uniformly convex if and only if $\lim _{\beta \rightarrow 1}(1-\beta) \xi(\beta)=0$,

(g) $X$ is uniformly smooth if and only if $\xi^{\prime}(0)=0$,

(h) $\xi_{X^{*}}(\beta)=1 / \xi^{-1}(1 / \beta)$ for $\beta \in[0,1)$,

(i) if $\xi(\beta)<1 /(1-\beta)$ for some $\beta$, then $X$ has uniformly normal structure.

The proof of these properties can be found in $[1,7]$ and also some of them as well as a more geometrical characterization of $\xi$ in [9-11].

Observe in particular that the behaviour of $\xi$ near 1 is related to convexity, and its behaviour near zero is related to smoothness.

The question of the existence of a sensible localization of the modulus of squareness was posed in [1]. In order to answer this question we define two new moduli.

From now on and for the sake of clarity, for any norm one vector $x, \lambda>0$ and $y$ with $\|y\|<1$, we put

$$
\omega_{x}(\lambda, y)=\omega((1+\lambda) x, y) \quad \text { and } \quad z_{x}(\lambda, y)=z((1+\lambda) x, y) .
$$

Therefore $\omega_{x}(\lambda, y)=\left\|(1+\lambda) x-z_{x}(\lambda, y)\right\| / \lambda$. Moreover, we can deduce that for $y \in \operatorname{span}\{x\}$ and for any $\lambda>0, \omega_{x}(\lambda, y)=1$, since $z_{x}(\lambda, y)$ would be $x$.

Definition 1.2. For any norm one vectors $x, y$ the pointwise modulus of squareness at $x$ in direction $y$ is the function $\xi_{X, x, y}=\xi_{x, y}:[0,1) \rightarrow[1, \infty)$ defined by

$$
\xi_{x, y}(\beta)=\sup \left\{\omega_{x}(\lambda, \gamma y):|\gamma| \leq \beta, \lambda>0\right\} .
$$

DEFINITION 1.3. For any norm one vector $x$ the local modulus of squareness at $x$ is the function $\xi_{X, x}=\xi_{x}:[0,1) \rightarrow[1, \infty)$ defined by

$$
\xi_{x}(\beta)=\sup \left\{\omega_{x}(\lambda, y):\|y\| \leq \beta, \lambda>0\right\}=\sup _{\|y\|=1}\left\{\xi_{x, y}(\beta)\right\} .
$$

Observe that for any subspace $M \subset X$ of dimension 2 containing norm one vectors $x, y$ we have $\xi_{x, y}=\xi_{M, x, y}$. For $\xi_{x}$ we establish an analogue to (a) of Theorem 1.1. Indeed,

$$
\xi_{x}(\beta)=\sup \left\{\xi_{M, x}(\beta): x \in M \subset X, \operatorname{dim} M=2\right\} .
$$

One can see that for any $\beta \in[0,1)$,

$$
\xi(\beta)=\sup \left\{\xi_{x}(\beta): x \in S_{X}\right\}=\sup \left\{\xi_{x, y}(\beta): x, y \in S_{X}\right\} .
$$

We shall show how these moduli are related to various geometrical properties of the norm of $X$. In particular, in Section 3 we recall the notions of Gâteaux smoothness and Fréchet smoothness and show that whether or not a normed space $X$ is Fréchet (resp. Gâteaux) smooth depends on the 
behaviour of the local (resp. pointwise) modulus of squareness near zero. In Section 4 we recall the notions of local uniform convexity and strict convexity and show that whether or not $X$ is locally uniformly (resp. strictly) convex depends on the behaviour of the local (resp. pointwise) modulus of squareness near 1. More precisely, we shall establish:

THEOREM 1.4. Let $X$ be a normed space and $x$ a norm one vector. Then

(a) $X$ is Gâteaux smooth at $x$ iff $\xi_{x, y}^{\prime}(0)=0$ for all $y$ with $\|y\|=1$.

(b) $X$ is Fréchet smooth at $x$ iff $\xi_{x}^{\prime}(0)=0$.

(c) $X$ is strictly convex at $x$ iff $\lim _{\beta \rightarrow 1}(1-\beta) \xi_{x, y}(\beta)=0$ for all $y$ with $\|y\|=1$.

(d) $X$ is locally uniformly convex at $x$ iff $\lim _{\beta \rightarrow 1}(1-\beta) \xi_{x}(\beta)=0$.

In the following section we focus on the properties of the ratio $\omega_{x}(\cdot, \cdot)$.

2. Properties of $\omega_{x}(\lambda, y)$. By a normed space we mean a pair $(X,\|\cdot\|)$, where $X$ is a linear space and $\|\cdot\|$ is a norm, although we will often write $X$ instead of $(X,\|\cdot\|)$. We set $B_{X}=\{x \in X:\|x\| \leq 1\}$ and $S_{X}=\{x \in X$ : $\|x\|=1\}$.

The following lemma can be found in [1] as part of the proof that $\xi$ is locally Lipschitz continuous.

Lemma 2.1. Let $X$ be a normed space and $x, y \in S_{X}$. Then, for any $\lambda>0$ and $0 \leq \beta<\gamma<1$,

$$
\omega_{x}(\lambda, \gamma y)-\omega_{x}(\lambda, \beta y) \leq \xi_{1}(\gamma)-\xi_{1}(\beta) .
$$

For fixed norm one vectors $x, y$, the modulus $\xi_{x, y}$ can be expressed in a simpler way:

Proposition 2.2. Let $X$ be a normed space and $x, y$ two norm one vectors. Then, for all $\beta \in[0,1)$,

$$
\xi_{x, y}(\beta)=\sup \left\{\omega_{x}(\lambda, \pm \beta y): \lambda>0\right\} .
$$

Proof. It is enough to show that for any fixed $\lambda>0$ and any $\gamma \leq \beta$ we have $\omega_{x}(\lambda, \beta y) \geq \omega_{x}(\lambda, \gamma y)$. We use the following result which can be found in $[3,4,8]$.

LEMma 2.3. Let $X$ be a two-dimensional normed space and let $K_{1}, K_{2}$ be closed convex subsets of $X$ with nonempty interior. If $K_{1} \subset K_{2}$ then $r\left(K_{1}\right) \leq r\left(K_{2}\right)$, where $r\left(K_{i}\right)$ denotes the length of the circumference of $K_{i}$, $i=1,2$.

This lemma can be applied to the triangles: $K_{1}$ with vertices the origin, $z_{x}(\lambda, \gamma y)$ and $(1+\lambda) x$, and $K_{2}$ with vertices the origin, $z_{x}(\lambda, \beta y)$ and $(1+\lambda) x$. 
Therefore

$$
\begin{aligned}
r\left(K_{1}\right) & =\|(1+\lambda) x\|+\left\|z_{x}(\lambda, \gamma y)\right\|+\left\|(1+\lambda) x-z_{x}(\lambda, \gamma y)\right\| \\
& \leq\|(1+\lambda) x\|+\left\|z_{x}(\lambda, \beta y)\right\|+\left\|(1+\lambda) x-z_{x}(\lambda, \beta y)\right\|=r\left(K_{2}\right) .
\end{aligned}
$$

Simplifying and dividing by $\lambda$, we obtain the desired inequality.

Proposition 2.4. Let $X$ be a normed space. If $x, y$ are norm one vectors and $0 \leq \beta<\gamma<1$, then

$$
\begin{aligned}
\xi_{x, y}(\gamma)-\xi_{x, y}(\beta) & \leq \xi_{1}(\gamma)-\xi_{1}(\beta), \\
\xi_{x}(\gamma)-\xi_{x}(\beta) & \leq \xi_{1}(\gamma)-\xi_{1}(\beta) .
\end{aligned}
$$

In particular, $\xi_{x, y}$ and $\xi_{x}$ are locally Lipschitz continuous functions.

Proof. From Lemma 2.1 we deduce that $\omega_{x}(\lambda, \gamma y)-\xi_{x, y}(\beta) \leq \xi_{1}(\gamma)-$ $\xi_{1}(\beta)$ and, by Proposition 2.2, we obtain inequality (2.1), taking suprema over $\lambda>0$. Inequality (2.2) follows similarly from (2.1), on taking suprema over $y \in S_{X}$.

Trying to simplify the expression for $\xi_{x, y}$ obtained in Proposition 2.2, one can study the behaviour of the function $\omega_{x}(\cdot, y)$ for fixed $x \in S_{X}$ and $y \in \stackrel{\circ}{B}_{X}$. The next useful result is evident.

Proposition 2.5. Let $X$ be a normed space and $x \in S_{X}$. Then

$$
1 \leq \omega_{x}(\lambda):=\sup \left\{\omega_{x}(\lambda, y): y \in \stackrel{\circ}{B}_{X}\right\} \leq 1+2 / \lambda .
$$

We now prove that the limit of the function $\omega_{x}(\lambda, y)$ when $\lambda$ goes to zero always exists and we compute it.

Recall that for a normed space $X$ and $x, y \in X \backslash\{0\}$, one can define the right derivative of the norm at $x$ in direction $y$ as the limit

$$
N_{+}(x, y)=\lim _{\lambda \searrow 0} \frac{\|x+\lambda y\|-\|x\|}{\lambda} .
$$

Proposition 2.6. Let $X$ be any normed space, $x \in S_{X}$, and $y \in X$ with $\|y\|<1$. Then

$$
\lim _{\lambda \searrow 0} \omega_{x}(\lambda, y)=\frac{\|x-y\|}{1-N_{+}(x, y)} .
$$

In order to prove this result we need to introduce some notation.

Fix a normed space $X, x \in S_{X}$ and $y \in \stackrel{\circ}{B}_{X}$ with $y \notin \operatorname{span}\{x\}$. We denote by $z^{\prime}(\lambda)$ the unique vector which lies in $\operatorname{span}\left\{z_{x}(\lambda, y)\right\}$ and on the ray which starts at $x$ and has direction $y$, that is,

$$
z^{\prime}(\lambda)=\{x+\mu y: \mu \geq 0\} \cap \operatorname{span}\left\{z_{x}(\lambda, y)\right\} .
$$

We can write $z^{\prime}(\lambda)=x+\mu(\lambda) y$ for some $\mu(\lambda) \geq 0$. Denote by $f_{\lambda}$ a continuous functional on $X$ satisfying $f_{\lambda}(x)=f_{\lambda}\left(z_{x}(\lambda, y)\right)=1$. We can also write $z_{x}(\lambda, y)=(1+\lambda) x+\nu(\lambda)(y-(1+\lambda) x)$ for some $\nu(\lambda) \in[0,1]$. 
Lemma 2.7. Let $X$ be a normed space, $x \in S_{X}$ and $y \in \stackrel{\circ}{B}_{X}$ such that $y \notin \operatorname{span}\{x\}$. Then

(a) $\lim _{\lambda \searrow 0} z_{x}(\lambda, y)=x$.

(b) $\lim _{\lambda \searrow 0} \mu(\lambda)=0$.

(c) $\lim _{\lambda \searrow 0} f_{\lambda}(y)=N_{+}(x, y)$.

Proof of Lemma 2.7. For (a) it is enough to show that $\nu(\lambda)$ tends to zero as $\lambda \rightarrow 0$. First, observe that $\varphi(t)=\|(1+\lambda) x+t(y-(1+\lambda) x)\|$ is a convex function satisfying $\varphi(1)=\|y\|$ and $\varphi(0)=1+\lambda$. Therefore $\varphi(t) \leq(1+\lambda)+t(\|y\|-(1+\lambda))$ for $t \in[0,1]$. Secondly, since $z_{x}(\lambda, y) \in S_{X}$, we have $\varphi(\nu(\lambda))=1$, that is, $1 \leq(1+\lambda)+\nu(\lambda)(\|y\|-(1+\lambda))$. Finally, since $\nu(\lambda) \in[0,1]$, we obtain $\lim _{\lambda \searrow 0} \nu(\lambda)=0$ and $(\mathrm{a})$ is proved.

For $(\mathrm{b})$, observe that $z_{x}(\lambda, y)=(1+\lambda)(1-\nu(\lambda)) x+\nu(\lambda) y$. Since $z^{\prime}(\lambda)$ lies in $\operatorname{span}\left\{z_{x}(\lambda, y)\right\}$, there exists $\alpha(\lambda) \in \mathbb{R}$ such that

$$
x+\mu(\lambda) y=z^{\prime}(\lambda)=\alpha(\lambda) z_{x}(\lambda, y),
$$

from which $\alpha(\lambda)=(1+\lambda)^{-1}\left(1-\nu_{x}(\lambda)\right)^{-1}$ and then

$$
\mu(\lambda)=\nu(\lambda) /[(1+\lambda)(1-\nu(\lambda))]
$$

Since $\nu(\lambda)$ converges to 0 as $\lambda \rightarrow 0$, (b) is proved.

In order to show (c), observe that, by (b), we have

$$
N_{+}(x, y)=\lim _{\lambda \searrow 0} \frac{\|x+\mu(\lambda) y\|-\|x\|}{\mu(\lambda)}=\lim _{\lambda \searrow 0} \frac{\left\|z^{\prime}(\lambda)\right\|-\|x\|}{\mu(\lambda)} .
$$

Since $z^{\prime}(\lambda) \in \operatorname{span}\{z\},\left\|z^{\prime}(\lambda)\right\|=f_{\lambda}\left(z^{\prime}(\lambda)\right)$. Hence, as $f_{\lambda}(x)=\|x\|$,

$$
N_{+}(x, y)=\lim _{\lambda \searrow 0} \frac{f_{\lambda}\left(z^{\prime}(\lambda)\right)-f_{\lambda}(x)}{\mu(\lambda)}=\lim _{\lambda \searrow 0} \frac{\mu(\lambda) f_{\lambda}(y)}{\mu(\lambda)}=\lim _{\lambda \searrow 0} f_{\lambda}(y) .
$$

Proof of Proposition 2.6. First of all, if $y \in \operatorname{span}\{x\}$ then $1-N_{+}(x, y)=$ $\|x-y\|$, and since $\omega_{x}(\lambda, y)=1$, this case is clear. So, assume that $y \notin \operatorname{span}\{x\}$ and consider the unique vector $w(\lambda)$ satisfying the conditions $f_{\lambda}(w(\lambda))=1$ and $w(\lambda) \in\{\mu((1+\lambda) x-y): \mu \geq 0\}$. One can easily see, by comparing similar triangles, that $\omega_{x}(\lambda, y)=\|w(\lambda)\|$. Since $f_{\lambda}(w(\lambda))=1$, it is clear that

$$
w(\lambda)=\left(1+\lambda-f_{\lambda}(y)\right)^{-1}[(1+\lambda) x-y]
$$

that is,

$$
\omega_{x}(\lambda, y)=\frac{\|(1+\lambda) x-y\|}{1+\lambda-f_{\lambda}(y)} .
$$

Using the continuity of the norm and item (c) of the previous lemma we obtain the desired equality.

REMARK 2.8. However, this last fact does not help to compute $\xi_{x, y}(\beta)$, since the function $\omega_{x}(\cdot, y)$ is neither convex nor monotonic as the following example shows. 
EXAmple 2.9. For any $0<\varepsilon<1 / 2$, consider in $\mathbb{R}^{2}$ the norm defined by

$$
\|x\|=\max \left\{(1-\varepsilon)^{-1}\|x\|_{\infty},\|x\|_{1}\right\},
$$

and the vectors $x=(1-\varepsilon, 0)$ and $y=(\varepsilon, 1-\varepsilon)$. Fix $\beta \geq 1-\varepsilon$. Here is the graph of the function $\omega_{x}(\cdot, \beta y)$ for $\varepsilon=0.2$ and $\beta=0.88$.

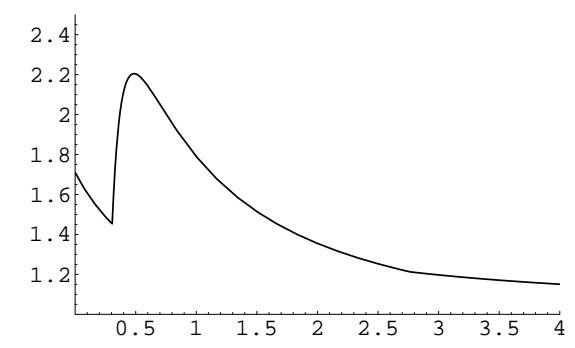

Fig. 1

3. On differentiability and localized squareness moduli. Throughout this section $X$ will be a normed space endowed with the norm $\|\cdot\|$. The collection of support functionals for a norm one vector $x$ is defined as

$$
\mathcal{D}(x)=\left\{f \in X^{*}:\|f\|=1, f(x)=\|x\|=1\right\} .
$$

We recall that the modulus of smoothness of a normed space is the function $\varrho:[0, \infty) \rightarrow \mathbb{R}^{+}$defined by

$$
\varrho(\beta)=\sup \{(\|x+\beta y\|+\|x-\beta y\|) / 2-1:\|x\|=\|y\|=1\} .
$$

Localizations of this modulus are the local modulus of smoothness, defined for any $x \in S_{X}$ and all $\beta \in[0, \infty)$ by

$$
\varrho_{x}(\beta)=\sup \{(\|x+\beta y\|+\|x-\beta y\|) / 2-1:\|y\|=1\},
$$

and the pointwise modulus of smoothness, defined for any norm one vectors $x, y$ and all $\beta \in[0, \infty)$ by

$$
\varrho_{x, y}(\beta)=(\|x+\beta y\|+\|x-\beta y\|) / 2-1 .
$$

Recall that a normed space is: Gâteaux smooth at $x \in S_{X}$ in direction $y \in S_{X}$ iff $\varrho_{x, y}(\beta) / \beta \rightarrow 0$ as $\beta \rightarrow 0 ;$ Gâteaux smooth at $x \in S_{X}$ iff it is Gâteaux smooth at $x$ in every direction $y \in S_{X}$; Gâteaux smooth iff it is Gâteaux smooth at any $x \in S_{X}$; Fréchet smooth at $x \in S_{X}$ iff $\varrho_{x}(\beta) / \beta \rightarrow 0$ as $\beta \rightarrow 0$; and Fréchet smooth iff it is Fréchet smooth at any $x \in S_{X}$. 
For any norm one vectors $x, y$, we define the function $\varepsilon_{x, y}:[0, \infty) \rightarrow$ $[0, \infty)$ by the formula

$$
\varepsilon_{x, y}(\beta)=\sup \left\{\frac{\|x+\beta w\|-\|x\|}{\beta}-f(w): w \in Y, f \in \mathcal{D}_{Y}(x)\right\},
$$

where $Y=\operatorname{span}\{x, y\}$ and $\mathcal{D}_{Y}(x)=\left\{\left.f\right|_{Y}: f \in \mathcal{D}(x)\right\}$. One can observe that this function is increasing and that the space is Gâteaux smooth at $x$ in direction $y$ if and only if $\varepsilon_{x, y}(\beta) \rightarrow 0$ as $\beta \rightarrow 0$. Let us show a relation between $\varepsilon_{x, y}$ and the pointwise modulus of squareness $\xi_{x, y}$.

Proposition 3.1. For any norm one vectors $x, y$ and all $\beta \in[0,1)$,

$$
\xi_{x, y}(\beta) \leq 1+\frac{2 \beta}{(1-\beta)^{2}} \varepsilon_{x, y}\left(\frac{2 \beta}{1-\beta}\right) .
$$

Proof. Fix $x, y \in S_{X}, \lambda>0, \beta \in[0,1)$ and a linear functional $f \in \mathcal{D}_{Y}(x)$. Then there exists $z_{0} \in[\beta y,(1+\lambda) x]$ such that $f\left(z_{0}\right)=1$. Pick a vector $u$ such that $f(u)=0$ and $z_{0} \in[u,(1+\lambda) x]$. It follows that there exists $\mu \geq 0$ such that $u=(1-\mu)(1+\lambda) x+\mu \beta y$ and, since $f(u)=0$, that $\mu=(1+\lambda) /(1+\lambda-\beta f(y))$. Thus,

$$
\|u\| \leq \frac{(1+\lambda) \beta}{1+\lambda-\beta f(y)}(|f(y)|+1) \leq \frac{2 \beta}{1-\beta} .
$$

As $z_{0} \in[u,(1+\lambda) x]$, there exists $\alpha \in(0,1)$ such that $z_{0}=(1-\alpha)(1+\lambda) x$ $+\alpha u$. Using the fact that $f\left(z_{0}\right)=1$, it is easily seen that $\alpha=\lambda /(1+\lambda)$. Therefore

$$
\begin{aligned}
& \frac{\left\|z_{0}-x\right\|}{\lambda}=\frac{\|u\|}{1+\lambda} \leq\|u\| \leq \frac{2 \beta}{1-\beta}, \\
& \left\|z_{0}-x\right\|=\frac{\lambda}{1+\lambda}\|u\| \leq\|u\| \leq \frac{2 \beta}{1-\beta} .
\end{aligned}
$$

Observe now that, from the definition of $\varepsilon_{x, y}$, it follows that

$$
\left\|(1+\lambda) x-z_{0}\right\|-\|\lambda x\| \leq\left\|x-z_{0}\right\| \varepsilon_{x, y}\left(\left\|x-z_{0}\right\| / \lambda\right) .
$$

Dividing by $\lambda$ and using (3.1) one obtains the inequality

$$
\frac{\left\|(1+\lambda) x-z_{0}\right\|}{\lambda} \leq 1+\frac{2 \beta}{1-\beta} \varepsilon_{x, y}\left(\frac{2 \beta}{1-\beta}\right) .
$$

Now, put $z=z_{x}(\lambda, \beta y)$ and denote by $\xi_{X}$ the modulus of squareness of $X$. One can easily see that $\left\|z-z_{0}\right\| \leq\left(\left\|z_{0}\right\|-1\right) \xi_{X}(\beta)$ and $\left\|z_{0}\right\|-1 \leq$ $\left\|x-z_{0}\right\| \varepsilon_{x, y}\left(\left\|x-z_{0}\right\|\right)$. Putting both together, and using (3.1), (3.2) and $\xi_{X} \leq \xi_{1}$, one has

$$
\frac{\left\|z-z_{0}\right\|}{\lambda} \leq \xi_{1}(\beta)\left(\frac{2 \beta}{1-\beta}\right) \varepsilon_{x, y}\left(\frac{2 \beta}{1-\beta}\right) .
$$


Finally, since

$$
\omega_{x}(\lambda, \beta y) \leq \frac{\left\|(1+\lambda) x-z_{0}\right\|}{\lambda}+\frac{\left\|z-z_{0}\right\|}{\lambda},
$$

using (3.3) and (3.4) one obtains

$$
\omega_{x}(\lambda, \beta y) \leq 1+\frac{2 \beta}{1-\beta} \varepsilon_{x, y}\left(\frac{2 \beta}{1-\beta}\right)\left(1+\xi_{1}(\beta)\right),
$$

which, on taking suprema over $\lambda>0$, finishes the proof.

Now we establish a relation between the pointwise modulus of squareness $\xi_{x, y}$ and the pointwise modulus of smoothness $\varrho_{x, y}$.

Proposition 3.2. For any norm one vectors $x, y$ and for every $\beta \in$ $[0,1)$,

$$
\begin{gathered}
\varrho_{x, y}(\beta) \leq \xi_{x, y}(\beta)-1, \\
\varrho_{x}(\beta) \leq \xi_{x}(\beta)-1 .
\end{gathered}
$$

Proof. Observe that the second inequality follows from the first on taking suprema over $y \in S_{X}$. Therefore we just have to show (3.5). Fix norm one vectors $x, y$. For a fixed $\beta \in[0,1)$ and $\lambda>0$, we set

$$
\begin{gathered}
y_{1}=y_{1}(\lambda, \beta y)=-(1+\lambda) \beta y, \quad y_{2}=y_{2}(\lambda, \beta y)=(1+\lambda) \beta y, \\
x^{\prime}=(1+\lambda) x, \quad z_{i}=\left(1-\alpha_{i}\right) x^{\prime}+\alpha_{i} y_{i},
\end{gathered}
$$

where $\alpha_{i} \in[0,1]$ for $i=1,2$.

On one hand, $1=\left\|z_{i}\right\| \geq f\left(z_{i}\right)$ for any $f \in \mathcal{D}(x)$. Therefore $\alpha_{i} \geq$ $\lambda /\left(1+\lambda-f\left(y_{i}\right)\right)$. On the other hand, $\left\|x^{\prime}-y_{i}\right\|=(1+\lambda)\|x \pm \beta y\|$. Since, for $\lambda<(1-\beta) / \beta$,

$$
\frac{\alpha_{i}(\lambda)\left\|x^{\prime}-y_{i}\right\|}{\lambda}=\omega_{x}(\lambda, \pm(1+\lambda) \beta y) \leq \xi_{x, y}((1+\lambda) \beta),
$$

we have

$$
\left\|x^{\prime}-y_{1}\right\|+\left\|x^{\prime}-y_{2}\right\| \leq \xi_{x, y}((1+\lambda) \beta)\left(\frac{\lambda}{\alpha_{1}}+\frac{\lambda}{\alpha_{2}}\right) .
$$

Since $\alpha_{i} \geq \lambda /\left(1+\lambda-f\left(y_{i}\right)\right)$ we deduce that

$$
\begin{aligned}
\left\|x^{\prime}-y_{1}\right\|+\left\|x^{\prime}-y_{2}\right\| & \leq \xi_{x, y}((1+\lambda) \beta)\left(2+2 \lambda-\left(f\left(y_{1}\right)+f\left(y_{2}\right)\right)\right) \\
& =\xi_{x, y}((1+\lambda) \beta)(2+2 \lambda)=2 \xi_{x, y}((1+\lambda) \beta)(1+\lambda),
\end{aligned}
$$

and therefore

$$
\|x+\beta y\|+\|x-\beta y\| \leq \frac{\left\|x^{\prime}-y_{1}\right\|+\left\|x^{\prime}-y_{2}\right\|}{1+\lambda} \leq 2 \xi_{x, y}((1+\lambda) \beta),
$$

which means that

$$
\varrho_{x, y}(\beta) \leq \xi_{x, y}((1+\lambda) \beta)-1
$$


Since this is true for $\lambda<(1-\beta) / \beta$, we can let $\lambda$ tend to 0 and, by the continuity of $\xi_{x, y}$, we obtain the desired inequality.

TheOREm 3.3. Let $\xi_{x}$ and $\xi_{x, y}$ be the localized squareness moduli of $X$. Then

(a) $X$ is Gâteaux smooth at $x \in S_{X}$ in direction $y \in S_{X}$ if and only if $\xi_{x, y}^{\prime}(0)=0$

(b) $X$ is Gâteaux smooth at $x \in S_{X}$ if and only if $\xi_{x, y}^{\prime}(0)=0$ for all $y \in S_{X}$.

(c) $X$ is Gâteaux smooth if and only if $\xi_{x, y}^{\prime}(0)=0$ for all $x, y \in S_{X}$.

(d) $X$ is Fréchet smooth at $x \in S_{X}$ if and only if $\xi_{x}^{\prime}(0)=0$.

(e) $X$ is Fréchet smooth if and only if $\xi_{x}^{\prime}(0)=0$ for all $x \in S_{X}$.

Proof. (a) First, by inequality (3.5) of Proposition 3.2, it is straightforward that if $\xi_{x, y}^{\prime}(0)=0$ then $\varrho_{x, y}(\beta) / \beta$ tends to 0 as $\beta \rightarrow 0$, i.e. the norm is differentiable at $x$ in direction $y$.

Secondly, assume that $X$ is Gâteaux smooth at $x$ in direction $y$. If $x$ and $y$ are linearly dependent the result is trivial. Suppose then that $x$ and $y$ are linearly independent; then applying Proposition 3.1 one has

$$
\frac{\xi_{x, y}(\beta)-1}{\beta} \leq \frac{2}{(1-\beta)^{2}} \varepsilon_{x, y}\left(\frac{2 \beta}{1-\beta}\right) .
$$

Since the norm of $X$ is Gâteaux smooth at $x$ in direction $y$, we have $\varepsilon_{x, y}(t)$ $\rightarrow 0$ as $t \rightarrow 0$. This implies that $\xi_{x, y}^{\prime}(0)=0$.

(b) This follows from (a) since for convex functions the existence of all directional derivatives at $x$ implies Gâteaux smoothness at $x$.

(c) Evident from (b).

(d) On one hand, by inequality (3.6) of Proposition 3.2, it is clear that if $\xi_{x}^{\prime}(0)=0$ then $\varrho_{x}(\beta) / \beta$ tends to 0 as $\beta \rightarrow 0$, i.e. the space is Fréchet smooth at $x$.

On the other hand, if we assume that $X$ is Fréchet smooth at $x$, then applying Proposition 3.1, for any $y \in S_{X}$ we have

$$
\frac{\xi_{x, y}(\beta)-1}{\beta} \leq \frac{2}{(1-\beta)^{2}} \varepsilon_{x, y}\left(\frac{2 \beta}{1-\beta}\right) .
$$

Taking suprema over $y \in S_{X}$ we obtain

$$
\frac{\xi_{x}(\beta)-1}{\beta} \leq \frac{2}{(1-\beta)^{2}} \sup _{y \in S_{X}}\left\{\varepsilon_{x, y}\left(\frac{2 \beta}{1-\beta}\right)\right\} .
$$

Since the space is Fréchet smooth at $x$, the right-hand side tends to 0 as $\beta \rightarrow 0$. Therefore $\xi_{x}^{\prime}(0)=0$.

(e) This follows from (d). 
4. On convexity and localized squareness moduli. This section is devoted to showing a relation between the behaviour of the localized moduli of squareness near 1 and the convexity properties of a normed space $X$. In the first subsection the local modulus of squareness $\xi_{x}$ is related to local uniform convexity, and in the second subsection the pointwise modulus of squareness $\xi_{x, y}$ is related to strict convexity.

4.1. Local uniform convexity. Fix a normed space $X$ and $x \in S_{X}$. The space $X$ is said to be locally uniformly convex at $x$ if its local modulus of convexity

$$
\delta_{x}(\varepsilon)=\inf \left\{1-\left\|\frac{x+y}{2}\right\|:\|y\|=1,\|x-y\| \geq \varepsilon\right\}
$$

is strictly positive for each $\varepsilon>0$. The number $\varepsilon_{0}(x)=\sup \left\{\varepsilon: \delta_{x}(\varepsilon)=0\right\}$ will be called the characteristic of convexity at $x$. Obviously, $X$ is locally uniformly convex at $x$ if and only if $\varepsilon_{0}(x)=0$.

One calls $D(x, \beta)=\operatorname{co}\left(\{x\} \cup \beta B_{X}\right)$ the drop of $\beta B_{X}$ with respect to the point $x$, and $R(x, \beta)=D(x, \beta) \backslash \beta B_{X}$ the residue. In [1] the authors observe that $X$ is locally uniformly convex at $x$ iff $\operatorname{diam} R(x, \beta) \rightarrow 0$ as $\beta \rightarrow 0$.

Recall that the radius of a set $A$ relative to a point $x$ is defined by $\operatorname{rad}(x, A)=\sup _{a \in A}\|x-a\|$. It is clear that $\operatorname{diam}(A) / 2 \leq \operatorname{rad}(x, A) \leq$ $\operatorname{diam}(A)$ whenever $x \in A$. For $\|x\|=1$ and $0<\beta<1$, Kadets [6] defined the set $G(x, \beta)=\left\{y:[y, z] \subset B_{X} \backslash \beta \stackrel{B}{B}_{X}\right\}$, and noted that $X$ is locally uniformly convex at $x$ iff $\operatorname{rad}(x, G(x, \beta)) \rightarrow 0$ as $\beta \rightarrow 1$. Moreover, it is known that the function $\epsilon(x, \beta)=\operatorname{rad}(x, G(x, \beta))$ is uniformly continuous on the set $S_{X} \times[0, r]$ for all $r<1$ and that $\epsilon$ is continuous at $(x, 1)$ if the norm is locally uniformly convex at $x \in S_{X}$ (see $[2,5]$ ).

It is also well known that the norm is locally uniformly convex at $x$ if and only if whenever a sequence $\left\{x_{n}\right\}_{n}$ satisfies

$$
\lim _{n \rightarrow \infty}\left(2\left(\|x\|^{2}+\left\|x_{n}\right\|^{2}\right)-\left\|x+x_{n}\right\|^{2}\right)=0,
$$

then $\lim _{n}\left\|x_{n}-x\right\|=0$. This can be shown easily by using the local modulus of convexity defined above. Finally, we say that the norm of $X$ is locally uniformly convex if it is locally uniformly convex at all $x \in S_{X}$.

Lemma 4.1. If a normed space is locally uniformly convex at $x \in S_{X}$, then

$$
\lim _{\lambda \rightarrow 0} \sup _{y \in \dot{B}_{X}}\left\|x-z_{x}(\lambda, y)\right\|=0 .
$$

Proof. Observe that for any $\lambda>0$ and $y$ with $\|y\|<1$ all points of the segment $\left[(1+\lambda) x, z_{x}(\lambda, y)\right]$ different from $z_{x}(\lambda, y)$ are outside the closed unit ball. Indeed, the function $f(\alpha)=\|\alpha(1+\lambda) x+(1-\alpha) z(\lambda, y)\|$ satisfies $f(0)=1$ and there exists $\alpha_{0}<0$ such that $f\left(\alpha_{0}\right)=\|y\|<1$. Since $f$ is 
convex we obtain $f(\alpha)>1$ whenever $\alpha>0$. In particular,

$$
f(1 / 2)=\frac{1+\lambda}{2}\left\|x+\frac{z_{x}(\lambda, y)}{1+\lambda}\right\|>1 \text {. }
$$

Therefore,

$$
\begin{aligned}
0 \leq 2\|x\|^{2}+2\left\|\frac{z_{x}(\lambda, y)}{1+\lambda}\right\|^{2}-\left\|x+\frac{z_{x}(\lambda, y)}{1+\lambda}\right\|^{2} & <2+\frac{1}{(1+\lambda)^{2}}-\frac{4}{(1+\lambda)^{2}} \\
& =2-\frac{2}{(1+\lambda)^{2}}
\end{aligned}
$$

where the right hand side tends to 0 uniformly over all $y \in \stackrel{\circ}{B}_{X}$ and, since the space is locally uniformly convex at $x, z_{x}(\lambda, y)$ converges to $x$ uniformly in $y \in \stackrel{\circ}{B}_{X}$.

TheOREM 4.2. For any normed space $X$ and for any $x \in S_{X}$, the following are equivalent:

(a) $X$ is locally uniformly convex at $x$.

(b) $\operatorname{diam} G(x, \beta) \rightarrow 0$ as $\beta \rightarrow 1$.

(c) $\operatorname{diam} R(x, \beta) \rightarrow 0$ as $\beta \rightarrow 1$.

(d) $\lim \sup _{\beta \rightarrow 1}(1-\beta) \xi_{x}(\beta)=0$.

(e) $\liminf _{\beta \rightarrow 1}(1-\beta) \xi_{x}(\beta)=0$.

Moreover, $\lim \inf _{\beta \rightarrow 1}(1-\beta) \xi_{x}(\beta) \geq \varepsilon_{0}(x)$.

Proof. The equivalence between (a), (b) and (c) is known. We claim that for all $0 \leq \beta<1$,

$$
\varepsilon_{0}(x)-1+\beta \leq(1-\beta) \xi_{x}(\beta) .
$$

Letting $\beta \rightarrow 1$ proves the last assertion and $(\mathrm{e}) \Rightarrow(\mathrm{a})$.

Inequality (4.1) is trivial if $\varepsilon_{0}(x)=0$, so suppose that $X$ is not locally uniformly convex at $x$. This means that, given any $\lambda>0$, we can find a norm one vector $y$, at distance at least $\varepsilon_{0}(x)$ from $x$, and such that for all $\gamma, \mu \geq 0$,

$$
\left(1+\lambda^{2}\right)\|\gamma x+\mu y\| \geq \gamma+\mu .
$$

Set $x^{\prime}=(1+\lambda) x$ and $y^{\prime}=\beta y$, so that $\left\|x^{\prime}-y^{\prime}\right\| \geq \varepsilon_{0}(x)-\lambda-(1-\beta)$. Then $z=z_{x}\left(\lambda, y^{\prime}\right)=(1-\alpha) x^{\prime}+\alpha y^{\prime}$ must satisfy

$$
1=\|z\| \geq \frac{1+\lambda-\alpha(1+\lambda-\beta)}{1+\lambda^{2}} \quad \text { and so } \quad \alpha \geq \frac{\lambda-\lambda^{2}}{1+\lambda-\beta} .
$$

But then

$$
\frac{\left\|x^{\prime}-z\right\|}{\lambda}=\frac{\alpha\left\|x^{\prime}-y^{\prime}\right\|}{\lambda} \geq \frac{(1-\lambda)\left(\varepsilon_{0}(x)-\lambda-(1-\beta)\right)}{1+\lambda-\beta} .
$$

Letting $\lambda \rightarrow 0$, we see that $\xi_{x}(\beta) \geq\left(\varepsilon_{0}(x)-1+\beta\right) /(1-\beta)$, which is (4.1). 
It is obvious that (d) implies (e), so it only remains to show (a) $\Rightarrow(d)$. Pick sequences $\left\{\beta_{n}\right\}_{n}$ tending to $1,\left\{\delta_{n}\right\}_{n}$ tending to $0, \lambda_{n}>0$ and vectors $y_{n} \in \beta_{n} B_{X}$ such that

$$
\xi_{x}\left(\beta_{n}\right)<\omega_{x}\left(\lambda_{n}, y_{n}\right)+\delta_{n}
$$

We have to distinguish two cases:

(a) If $\liminf \inf _{n}>0$, Lemma 2.5 shows that $M=\sup _{n}\left\{\omega_{x}\left(\lambda_{n}\right)\right\}<\infty$ and so

$$
\xi_{x}\left(\beta_{n}\right)<\omega_{x}\left(\lambda_{n}, y_{n}\right)+\delta_{n} \leq \omega_{x}\left(\lambda_{n}\right)+\delta_{n} \leq M+\delta_{n} .
$$

Therefore,

$$
\limsup _{n \rightarrow \infty}\left(1-\beta_{n}\right) \xi_{x}\left(\beta_{n}\right) \leq \lim _{n \rightarrow \infty}\left(1-\beta_{n}\right)\left(M+\delta_{n}\right)=0 .
$$

(b) If $\liminf \operatorname{in}_{n} \lambda_{n}=0$, then we can assume, passing to a subsequence, that $\lambda_{n} \rightarrow 0$. If necessary we can choose $y_{n}^{\prime}$ in such a way that $\left\|y_{n}^{\prime}\right\|=\beta_{n}$ and $\left.y_{n}^{\prime} \in\left[y_{n},\left(1+\lambda_{n}\right) x\right] \cap G\left(z_{x}\left(\lambda_{n}, y_{n}\right), \beta_{n}\right)\right)$. Set

$$
z_{n}=z_{x}\left(\lambda_{n}, y_{n}\right)=\alpha_{n}\left(1+\lambda_{n}\right) x+\left(1-\alpha_{n}\right) y_{n}^{\prime} .
$$

Then $1=\left\|z_{n}\right\| \leq \alpha_{n}\left(1+\lambda_{n}\right)+\left(1-\alpha_{n}\right) \beta_{n}$, from which it follows that $\left(1-\alpha_{n}\right)\left(1-\beta_{n}\right) \leq \alpha_{n} \lambda_{n}$ and

$$
\begin{aligned}
\left(1-\alpha_{n}\right)\left(1-\beta_{n}\right) \omega_{x}\left(\lambda_{n}, y_{n}^{\prime}\right) & \leq \alpha_{n}\left\|\left(1+\lambda_{n}\right) x-z_{n}\right\|=\left(1-\alpha_{n}\right)\left\|y_{n}^{\prime}-z_{n}\right\| \\
& \leq\left(1-\alpha_{n}\right) \operatorname{rad}\left(z_{n}, G\left(z_{n}, \beta\right)\right) .
\end{aligned}
$$

That is, $\left(1-\beta_{n}\right) \omega_{x}\left(\lambda_{n}, y_{n}\right)=\left(1-\beta_{n}\right) \omega_{x}\left(\lambda_{n}, y_{n}^{\prime}\right) \leq \epsilon\left(z_{n}, \beta_{n}\right)$. Lemma 4.1 tells us that $z_{n}$ tends to $x$ and therefore, since $\epsilon(\cdot, \cdot)$ is continuous at $(x, 1)$, we have

$$
\begin{aligned}
\limsup _{n \rightarrow \infty}\left(1-\beta_{n}\right) \xi_{x}\left(\beta_{n}\right) & \leq \limsup _{n \rightarrow \infty}\left(1-\beta_{n}\right) \omega_{x}\left(\lambda_{n}, y_{n}\right) \\
& \leq \lim _{n \rightarrow \infty} \epsilon\left(z_{n}, \beta_{n}\right)=\epsilon(x, 1)=0,
\end{aligned}
$$

which is what we wanted to show.

This proposition yields a new characterization of local uniform convexity.

Corollary 4.3. For any normed space $X$ the following are equivalent:

(a) $X$ is locally uniformly convex.

(b) $\operatorname{diam} G(x, \beta) \rightarrow 0$ as $\beta \rightarrow 1$ for all $x \in S_{X}$.

(c) $\operatorname{diam} R(x, \beta) \rightarrow 0$ as $\beta \rightarrow 1$ for all $x \in S_{X}$.

(d) $\lim \sup _{\beta \rightarrow 1}(1-\beta) \xi_{x}(\beta)=0$ for all $x \in S_{X}$.

(e) $\liminf \inf _{\beta \rightarrow 1}(1-\beta) \xi_{x}(\beta)=0$ for all $x \in S_{X}$.

4.2. Strict convexity. Let $X$ be a normed space and $x, w \in S_{X}$. The norm of $X$ is said to be strictly convex at $x$ in direction $w$ if there is no proper segment included in the unit sphere starting at $x$ with direction $w$. Similarly, it is said to be strictly convex at $x$ if there is no proper segment included in 
the unit sphere starting at $x$ in any direction. $X$ is said to be strictly convex if it is strictly convex at all its norm one vectors. We define $\varepsilon_{0}(x, w)$ to be the supremum of $\varepsilon>0$ such that the segment $[x, x+\varepsilon w]$ or $[x, x-\varepsilon w]$ lies on the unit sphere. We also define $C_{x}^{w}=\left\{y \in S_{X}: \exists \lambda \in \mathbb{R}, y=x+\lambda w\right\}$.

Proposition 4.4. Let $X$ be a normed space and $x, w$ two norm one vectors. If $\liminf _{\beta \rightarrow 1}(1-\beta) \xi_{x, y}(\beta)=0$ for all $y \in C_{x}^{w}$, then $X$ is strictly convex at $x$ in direction $w$. Moreover,

$$
\sup _{y \in C_{x}^{w}} \liminf _{\beta \rightarrow 1}(1-\beta) \xi_{x, y}(\beta) \geq \varepsilon_{0}(x, w) .
$$

Proof. Assume that $X$ is not strictly convex at $x$ in direction $w$. This means that $\varepsilon_{0}(x, w)>0$, and that for any $\varepsilon_{0}(x, w)>\delta>0$ there exists $y \in C_{x}^{w}$ such that $\|y-x\| \geq \varepsilon_{0}(x, w)-\delta$. Write $z=z_{x}(\lambda, \beta y)$. There exists $\alpha \in[0,1]$ such that $z=(1-\alpha)(1+\lambda) x+\alpha \beta y$. Let us compute $\alpha$. Fix $f \in \mathcal{D}(x)$ such that $f([x, y])=1$. We have $1=f(z)=(1-\alpha)(1+\lambda)+\alpha \beta$. Therefore $\alpha=\lambda /(1+\lambda-\beta)$.

On the other hand,

$$
\|(1+\lambda) x-\beta y\| \geq\|x-y\|-\|\lambda x+(1-\beta) y\| \geq \varepsilon_{0}(x, w)-\delta-\lambda-(1-\beta) .
$$

Therefore,

$$
\xi_{x, y}(\beta) \geq \omega_{x}(\lambda, \beta y)=\alpha \frac{\|(1+\lambda) x-\beta y\|}{\lambda} \geq \frac{\varepsilon_{0}(x, w)-\delta-\lambda-(1-\beta)}{1+\lambda-\beta} .
$$

Letting $\lambda \rightarrow 0$, we obtain $(1-\beta) \xi_{x, y}(\beta) \geq \varepsilon_{0}(x, w)-\delta-(1-\beta)$. Therefore

$$
\liminf _{\beta \rightarrow 0}(1-\beta) \xi_{x, y}(\beta) \geq \varepsilon_{0}(x, w)-\delta .
$$

This implies that $\liminf _{\beta \rightarrow 0}(1-\beta) \xi_{x, y}(\beta)>0$, which shows the first and, whenever $\varepsilon_{0}(x, w)>0$, the second assertion of the theorem. The proof is finished, since the second assertion is clear when $\varepsilon_{0}(x, w)=0$.

ThEOREM 4.5. For any normed space $X$ and for any $x \in S_{X}$ the following are equivalent:

(a) $X$ is strictly convex at $x$.

(b) $\lim \sup _{\beta \rightarrow 1}(1-\beta) \xi_{x, y}(\beta)=0$ for all $y \in S_{X}$.

(c) $\liminf _{\beta \rightarrow 1}(1-\beta) \xi_{x, y}(\beta)=0$ for all $y \in S_{X}$.

Proof. The implication (b) $\Rightarrow(\mathrm{c})$ is evident. The implication (c) $\Rightarrow$ (a) follows from Proposition 4.4. In order to see $(\mathrm{a}) \Rightarrow(\mathrm{b})$, fix $y \in S_{X}$, and pick $\left\{\beta_{n}\right\}_{n}$ tending to $1,\left\{\delta_{n}\right\}_{n}$ tending to $0, \lambda_{n}>0$ and vectors $y_{n}=\gamma_{n} y \in \beta_{n} B_{X}$ such that

$$
\xi_{x, y}\left(\beta_{n}\right)<\omega_{x}\left(\lambda_{n}, y_{n}\right)+\delta_{n}
$$

We have to distinguish two cases: 
(a) If $\liminf \inf _{n} \lambda_{n}>0$, then Lemma 2.5 shows that $M=\sup _{n}\left\{\omega_{x}\left(\lambda_{n}\right)\right\}$ $<\infty$ and so

$$
\xi_{x, y}\left(\beta_{n}\right)<\omega_{x}\left(\lambda_{n}, y_{n}\right)+\delta_{n} \leq \omega_{x}\left(\lambda_{n}\right)+\delta_{n} \leq M+\delta_{n} .
$$

Therefore,

$$
\limsup _{n \rightarrow \infty}\left(1-\beta_{n}\right) \xi_{x, y}\left(\beta_{n}\right) \leq \lim _{n \rightarrow \infty}\left(1-\beta_{n}\right)\left(M+\delta_{n}\right)=0 .
$$

(b) If $\liminf { }_{n} \lambda_{n}=0$, we can assume, passing to a subsequence, that $\lambda_{n} \rightarrow 0$. If necessary we can choose $y_{n}^{\prime}$ such that $\left\|y_{n}^{\prime}\right\|=\beta_{n}$ and $y_{n}^{\prime} \in\left[y_{n}\right.$, $\left.\left.\left(1+\lambda_{n}\right) x\right] \cap G_{Y}\left(z_{x}\left(\lambda_{n}, y_{n}\right), \beta_{n}\right)\right)$, where $Y=\operatorname{span}\{x, y\}$. Write $z_{n}=z_{x}\left(\lambda_{n}, y_{n}\right)$ $=\alpha_{n}\left(1+\lambda_{n}\right) x+\left(1-\alpha_{n}\right) y_{n}^{\prime}$. Then $1=\left\|z_{n}\right\| \leq \alpha_{n}\left(1+\lambda_{n}\right)+\left(1-\alpha_{n}\right) \beta_{n}$, from which it follows that $\left(1-\alpha_{n}\right)\left(1-\beta_{n}\right) \leq \alpha_{n} \lambda_{n}$ and

$$
\begin{aligned}
\left(1-\alpha_{n}\right)\left(1-\beta_{n}\right) \omega_{x}\left(\lambda_{n}, y_{n}^{\prime}\right) & \leq \alpha_{n}\left\|\left(1+\lambda_{n}\right) x-z_{n}\right\|=\left(1-\alpha_{n}\right)\left\|y_{n}^{\prime}-z_{n}\right\| \\
& \leq\left(1-\alpha_{n}\right) \operatorname{rad}\left(z_{n}, G_{Y}\left(z_{n}, \beta\right)\right) .
\end{aligned}
$$

That is, $\left(1-\beta_{n}\right) \omega_{x}\left(\lambda_{n}, y_{n}\right)=\left(1-\beta_{n}\right) \omega_{x}\left(\lambda_{n}, y_{n}^{\prime}\right) \leq \epsilon_{Y}\left(z_{n}, \beta_{n}\right)$. Since $Y$ is locally uniformly convex at $x$, Lemma 4.1 tells us that $z_{n}$ tends to $x$ and therefore, since $\epsilon_{Y}(\cdot, \cdot)$ is continuous at $(x, 1)$, we have

$$
\begin{aligned}
\limsup _{n \rightarrow \infty}\left(1-\beta_{n}\right) \xi_{x, y}\left(\beta_{n}\right) & \leq \limsup _{n \rightarrow \infty}\left(1-\beta_{n}\right) \omega_{x}\left(\lambda_{n}, y_{n}\right) \\
& \leq \lim _{n \rightarrow \infty} \epsilon_{Y}\left(z_{n}, \beta_{n}\right)=\epsilon_{Y}(x, 1)=0,
\end{aligned}
$$

which is what we wanted to show.

From this theorem one can easily deduce the following one.

TheOREM 4.6. For any normed space $X$ the following are equivalent:

(a) $X$ is strictly convex.

(b) $\lim \sup _{\beta \rightarrow 1}(1-\beta) \xi_{x, y}(\beta)=0$ for all $x, y \in S_{X}$.

(c) $\lim \inf _{\beta \rightarrow 1}(1-\beta) \xi_{x, y}(\beta)=0$ for all $x, y \in S_{X}$.

Acknowledgments. The author wishes to express his gratitude to Professor R. Deville for helpful comments and suggestions which improved the content of this paper.

\section{References}

[1] C. Benítez, K. Przesławski, and D. Yost, A universal modulus for normed spaces, Studia Math. 127 (1998), 21-46.

[2] C. Bessaga and A. Pełczyński, Selected Topics in Infinite-Dimensional Topology, Monograf. Mat. 58, PWN-Polish Sci. Publ., Warszawa, 1975.

[3] H. Busemann, The Geometry of Geodesics, Academic Press, New York, 1955.

[4] J. Gao and K. Lau, On two classes of Banach spaces with uniform normal structure, Studia Math. 99 (1991), 41-56.

[5] A. J. Guirao, Topological classification of Banach spaces, Univ. of Murcia. 
[6] M. I. Kadec, A proof of the topological equivalence of all separable infinite-dimensional Banach spaces, Funktsional. Anal. i Prilozhen. 1 (1967), no. 1, 61-70 (in Russian).

[7] K. Przesławski and D. Yost, Lipschitz retracts, selectors, and extensions, Michigan Math. J. 42 (1995), 555-571.

[8] J. J. Schäffer, Geometry of Spheres in Normed Spaces, Lecture Notes in Pure Appl. Math. 20, Dekker, New York, 1976.

[9] I. Şerb, On the behaviour of the tangential modulus of a Banach space. I, Rev. Anal. Numér. Théor. Approx. 24 (1995), 241-248.

[10] - On the behaviour of the tangential modulus of a Banach space. II, Mathematica 38(61) (1996), 199-207.

[11] - A Day-Nordlander theorem for the tangential modulus of a normed space, J. Math. Anal. Appl. 209 (1997), 381-391.

Departamento de Matemáticas

Universidad de Murcia

30100 Espinardo (Murcia), Spain

E-mail: ajguirao@um.es

Received December 22, 2006

Revised version November 8, 2007 